\title{
Is It Wrong to Apply the Intrinsic Rate of Natural Increase to Individuals and Compare It among Genotypes?
}

\author{
Takahiro Miyo \\ 2-17-4 Misumi-cho, Higashimurayama-shi, Tokyo 189-0023, Japan \\ Email: takahiro_miyo@hotmail.com
}

How to cite this paper: Miyo, T. (2018) Is It Wrong to Apply the Intrinsic Rate of Natural Increase to Individuals and Compare It among Genotypes? Open Journal of Genetics, 8, 1-13.

https://doi.org/10.4236/ojgen.2018.81001

Received: January 8, 2018

Accepted: March 3, 2018

Published: March 6, 2018

Copyright $\odot 2018$ by author and Scientific Research Publishing Inc. This work is licensed under the Creative Commons Attribution International License (CC BY 4.0).

http://creativecommons.org/licenses/by/4.0/

\section{(c) (i) Open Access}

\begin{abstract}
In order to gain insights into the seasonal dynamics of genetic variation in insecticide resistance within a natural population of Drosophila melanogaster during population growth, which we considered the most important ecological factor there, we conducted a series of genetic analyses of resistance factors involved in that population and compared individual-based intrinsic rates of natural increase among resistance genotypes. However, some researchers have argued that it is a misconception to apply the intrinsic rate of natural increase to individuals, because it is a population parameter. We consider that their criticisms were incorrect. In this article, I described our research briefly and set forth the reasons why we conducted these studies.
\end{abstract}

\section{Keywords}

Drosophila melanogaster, Insecticide Resistance, Intrinsic Rate of Natural Increase, Natural Population

\section{Introduction}

We have conducted a series of studies concerning the relationship between resistance to organophosphate insecticides and fitness [1]-[6]. This is because we endeavored to unearth the mechanism explaining the two-consecutive-year observations that the levels of resistance to organophosphates decreased within one Japanese local population of Drosophila melanogaster (Meigen) in the fall, when population sizes increased drastically [7]. To unearth the mechanism, we attempted to evaluate the intrinsic rate of natural increase for each individual and compare it among genotypes resistant and susceptible to organophosphates, especially among genotypes at the acetylcholinesterase locus, and obtained results 
suggesting that the intrinsic rates of increase for resistant genotypes were lower than those for susceptible genotypes [5]. However, some researchers argued that it was a misconception to apply the intrinsic rate of natural increase to individuals, because it is a population parameter [8]. Is it conceptually wrong to apply the intrinsic rate of natural increase to individuals? We consider that their criticisms were incorrect, and would like to explain why we did so, after our research is described briefly.

\section{Katsunuma Population of D. melanogaster}

D. melanogaster is actually not recognized as a direct target of insecticide application in the field, but this organism has provided many valuable findings on the development of insecticide resistance as a model system [9]. Although we have investigated genetic variation in insecticide resistance within natural populations of this species at several locations [6] [10], we especially focused on a natural population of D. melanogaster at Katsunuma (Yamanashi Prefecture, Japan) as a model system, for the Katsunuma population is one of the well-studied natural populations of Drosophila in Japan [11].

Katsunuma is famous for its wine production, and many vineyards expand continuously at Katsunuma and its surrounding areas (Figure 1). In the fall, the fermentation season, a large population of $D$. melanogaster can be found on dropped grapes and the masses of squeezed grapes dumped in the vineyards in the process of wine production [7] [10]. Although Drosophila flies per se are not recognized as pest species there, it is likely that the Drosophila population is indirectly exposed to selection pressures by insecticides, mainly organophosphates, applied for other insect pests [10]. Therefore, it would be expected that genetic variation in resistance to insecticides within the Katsunuma population of $D$. melanogaster could be influenced by various factors, including ecological and

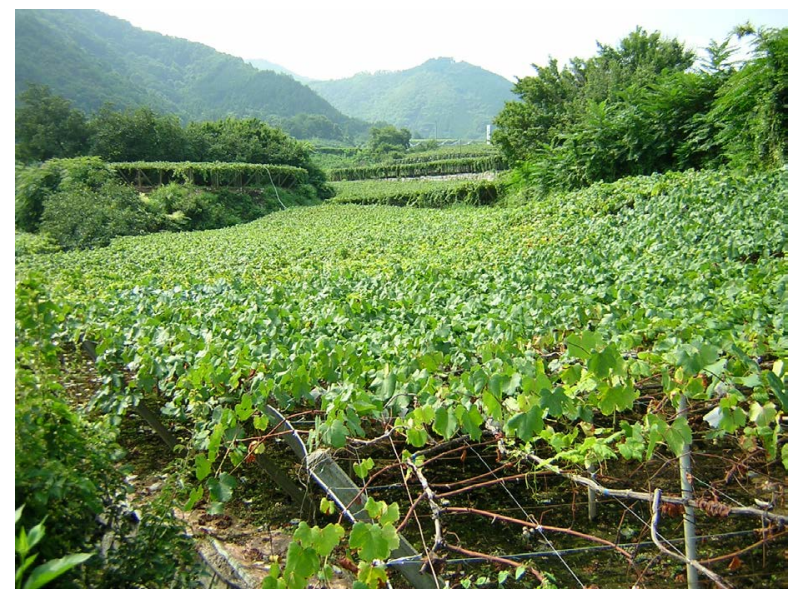

Figure 1. Vineyards at Katsunuma, Yamanashi Prefecture, Japan. Many vineyards expand at Katsunuma and its surrounding areas (taken by the author on August 25, 2006). We collected the natural population at Katsunuma four times, from each of which many isofemale lines were established and evaluated for susceptibility to insecticides. Detailed information is presented in [6] [7]. 
environmental factors as well as insecticides, and that a fluctuation occurred in genetic variation in resistance to one insecticide could affect simultaneously genetic variation in resistance to other insecticides through complex processes [7].

\section{Quantitative Analysis of Insecticide Resistance within the Katsunuma Population}

Establishing isofemale lines derived from females collected in the wild provides a basic approach for investigating genetic variation within natural populations of Drosophila [12]. Although this approach requires us to establish and maintain many isofemale lines from the same population in order to maintain genetic variation in the laboratory, there may be some advantages over other approaches. For example, this approach may avoid occasional loss of genetic variation under the laboratory condition, which may occur if mass populations suffer a bottleneck. This approach may also avoid accumulation of several resistance factors into every individual, which may occur if mass selection by an insecticide is performed, making results of genetic analyses of each resistance factor difficult to infer. In addition, this approach is relatively straightforward to conduct, though laborious, so that it can be effectively conducted for other insects which lack special genetic tools, such as balancer chromosomes in D. melanogaster.

After collecting the natural population of $D$. melanogaster at Katsunuma in the summer and fall of 1997 and 1998, we made 52 to 499 isofemale lines established in the laboratory. By using the filter-paper-contact method (see [7] [10] for details of the bioassay method and its condition), susceptibility to each of five insecticides was evaluated for each isofemale line from respective seasons (40 to 286 lines). The five insecticides used for evaluation were permethrin (pyrethroid), malathion, prothiophos, and fenitrothion (organophosphates), and DDT (organochlorine). Then, following the analysis of variance [12] [13], variance components, genetic variance $\left(V_{g}\right)$ and within-line variance $\left(V_{w}\right)$, were estimated, using the above mortality data sets [6] [14]. Figure 2 illustrates the results of quantitative analyses of mortality data obtained using the isofemale lines, which were arcsine-square-root transformed prior to the analyses. Although mortality of each isofemale line was evaluated with control replications (mean control mortality among lines for each season was <0.032; [6] [14]), and mean susceptibility was based on mortality data, of which adjustment using Abbott's formula [15] was indeed performed [7] [10], analyses of variance for estimating $V_{g}$ and $V_{w}$ were based on mortality data without adjustment, because control mortality could be largely due to environmental causes and/or experimental mishandling, so that it should be treated as within-line variation [6] [14]. Because $V_{W}$ components in susceptibility to each insecticide were almost the same magnitude among the seasons (Figure 2), $V_{g}$ components were likely to be based on experiments performed under reasonably controlled conditions (p. 339 in [16]). (Please note that variance in susceptibility to an insecticide is the same as variance in resistance to the insecticide, if resistance can be evaluated as survival, 

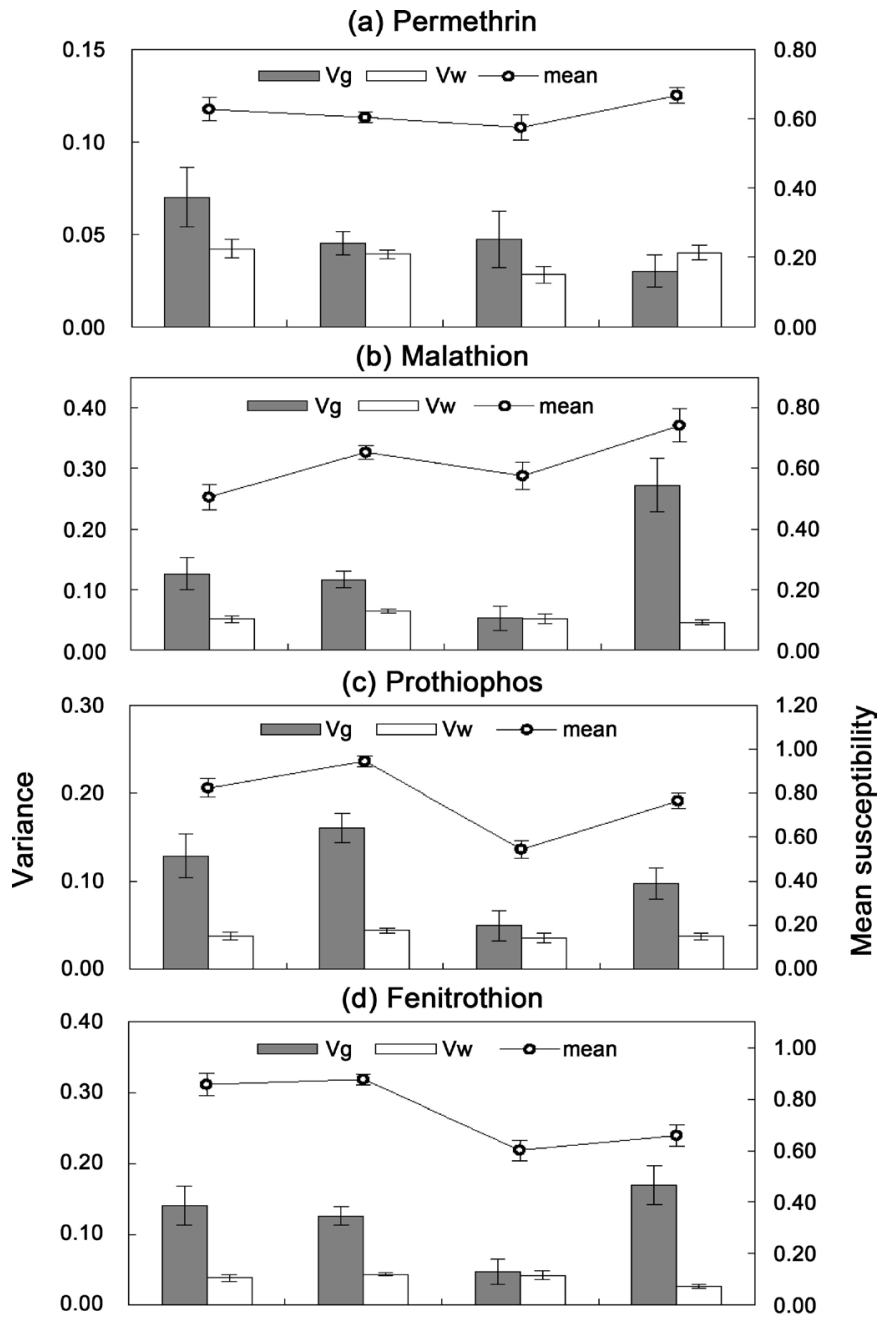

(e) DDT

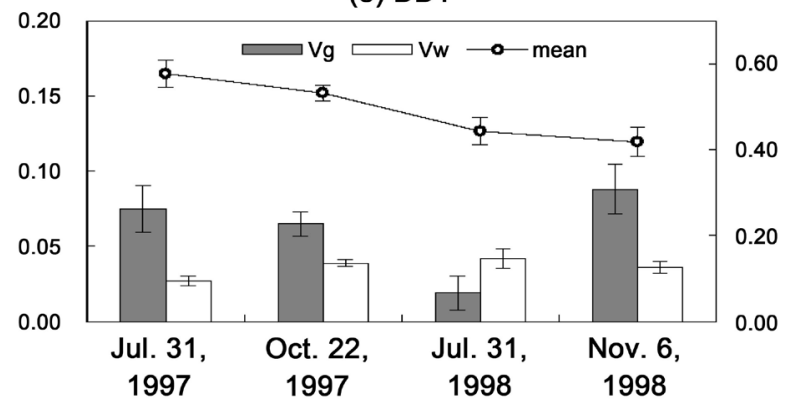

Collection date

Figure 2. Quantitative analyses of the dynamics of genetic variation in susceptibility to five insecticides within the Katsunuma population of D. melanogaster. (a) permethrin, (b) malathion, (c) prothiophos, (d) fenitrothion, (e) DDT. Error bars indicate standard errors of the means and variance component estimates. Variance estimates are from [6] [14], and mortality results are from [7].

because $\sin ^{-1} \sqrt{P}$ is equal to $\pi / 2-\sin ^{-1} \sqrt{1-P}$, where $P$ is mortality; therefore, $\left.V\left(\sin ^{-1} \sqrt{P}\right)=V\left(\pi / 2-\sin ^{-1} \sqrt{1-P}\right)=V\left(\sin ^{-1} \sqrt{1-P}\right)\right)$.

It was revealed that there existed significant genetic variation in susceptibility to each of the five insecticides, involving not only organophosphates but also 
permethrin (a pyrethroid) and DDT (an organochlorine), in the Katsunuma population of $D$. melanogaster (Figure 2). However, the behavior of genetic variation in susceptibility to each insecticide was different among insecticide classes. Figures 2(b)-(d) show seasonal fluctuations in susceptibility to organophosphates, in which the mean susceptibility to each of the three organophosphates tended to increase consistently in the fall for the two years, when the Katsunuma population of $D$. melanogaster increased. Contrary to this consistent tendency in mean, significant changes in $V_{g}$ in susceptibility to the three organophosphates were not observed between the summer and fall of 1997, but tended to increase in 1998. Unlike susceptibility to organophosphates, a significant tendency towards an increase in susceptibility was not observed in the fall in the cases of permethrin and DDT (Figure 2(a) and Figure 2(e)); however, the patterns of the fluctuation in $V_{g}$ were different between susceptibility to the two chemicals. These results suggest that genetic variation in susceptibility to insecticides within the Katsunuma population could not be explained by a simple mechanism, such that a single resistance factor showing cross-resistance to the five insecticides was involved in the Katsunuma population. (Please note that these mortality data were not obtained from one population cage, repeatedly bioassayed four times, but obtained from many isofemale lines, derived from females collected at Katsunuma in each season. Therefore, the possibility is unlikely that these fluctuations were caused by contamination of susceptible laboratory individuals.)

\section{Resistance Factors for Organophosphates within the Katsunuma Population}

Based on the results of mortality bioassays for isofemale lines, two resistant lines (\#609 and \#1465) and one susceptible line (\#451) were chosen to establish inbred lines for further genetic analyses [10]. Although the susceptible line might have slightly higher resistance levels than laboratory standard strains, we considered that susceptible lines derived from the Katsunuma population should be used, because we attempted to elucidate the dynamics of genetic variation in susceptibility to organophosphates within the Katsunuma population, and because laboratory strains usually used for susceptible standards did not exist in this population, so that they were not representatives of susceptible genotypes there.

Before examination, we are usually not sure how many resistance factors insect individuals possess, where these factors are located, and how large the effect of each factor is. Therefore, in the first place, chromosomal analyses of resistance to organophosphates were conducted, by using chromosome-substituted lines constructed between a resistant inbred \#1465-5 and a susceptible inbred \#451-10 [17] and between a resistant inbred \#609-10 and a susceptible inbred \#451-4 [3]. Chromosomal analyses of organophosphate resistance using chromosome-substituted lines between \#1465-5 and \#451-10 revealed that not only chromosome II but also chromosome III contributed to organophosphate resistance in this resistant line [17]. Therefore, genetic analyses for each factor should be conducted using 
chromosome-substituted lines, whose genetic backgrounds were replaced by those of the susceptible line, in order to make the effects of other resistance factors excluded as much as possible. Using chromosome-substituted lines, we then identified two resistance factors, one at $\sim$ II-62 and the other at $\sim$ III-50 [17]. In addition to the fact that the resistance factor on chromosome III was mapped near the acetylcholinesterase locus (Ace, III-52; [18]), the target-site for organophosphate and carbamate insecticides, the resistant individuals having chromosome III from \#1465-5 indeed showed 15 times higher $\mathrm{I}_{50}$ values (concentrations of chemicals that inhibit $50 \%$ of enzyme activity) for acetylcholinesterase (AChE) to fenitroxon (an organophosphate) than those from \#451-10, suggesting that the resistance factor on chromosome III was one of the mutated AChEs, and that there were not any factors on other chromosomes contributing to the AChE activity [4]. On the other hand, the resistance factor on chromosome II was suggested to be a member of the cytochrome P450 gene, according to results from inhibition assays using a synergist, piperonyl butoxide [4]. Therefore, it was suggested that one of the increased detoxification mechanisms as well as the target-site insensitivity contributed to resistance to organophosphates in the resistant line \#1465-5, and that these resistance factors constructed at least a part of genetic variation in resistance to organophosphates within the Katsunuma population of D. melanogaster [4]. However, the effects of each factor on resistance were not necessarily the same for the three organophosphates [17], so that the relative contributions of the two factors to genetic variation in resistance to organophosphates were expected to be different. A model system for evaluating the contributions of each resistance factor within the Katsunuma population suggested remarkably larger contributions of the resistant-type AChE to genetic variation especially in resistance to malathion and fenitrothion, relative to the cytochrome P450; therefore, it was expected that the change in the frequency of the resistant-type Ace gene would predominantly affect the dynamics of genetic variation in susceptibility to organophosphates in the case of the Katsunuma population [1].

In D. melanogaster, very useful genetic tools, such as balancer chromosomes, are available. Balancer chromosomes can be used for isolating chromosomes of wild-caught flies and making them homozygous [19]. We did not take advantage of the chromosome extraction technique using balancer chromosomes, but adopted the isofemale line approach in our studies. Because there were the relatively large effects of the resistant-type AChE on chromosome III in the case of the Katsunuma population [1], chromosome II extraction directly from wildcaught flies would have made results of genetic analyses difficult to infer, due to uncontrollable chromosome III effects, which would mask the effects of chromosome II. On the other hand, chromosome III extraction might have had little difficulties, but we had no information on the numbers, locations, and relative effects of resistance factors before examination, so that we think the isofemale line approach was appropriate for studying genetic variation in resistance to insecticides within the Katsunuma population of $D$. melanogaster at the stage 
where detailed information was not available.

\section{Comparison of Individual-Based Intrinsic Rates of Natural Increase among Resistance Genotypes}

Based on the quantitative analyses of susceptibility to insecticides within the Katsunuma population, seasonal fluctuations in susceptibility to organophosphates were observed for the two years, in which susceptibility levels for the three organophosphates tended to increase in the fall (Figures 2(b)-(d)). Because, contrary to the cases of organophosphates, susceptibility levels for DDT and permethrin showed a tendency towards rather a decrease in the fall (Figure 2(a) and Figure 2(e)), it was unlikely that the seasonal fluctuations in organophosphate resistance were caused by susceptible migrants moving into the Katsunuma population in the fall. We thus have analyzed the relationship between resistance to organophosphates and fitness components, from the standpoint that the resistance factor for the three organophosphates on chromosome III had disadvantageous effects on fitness, in a series of studies [2] [3] [5]. This is because reduction in levels of resistance to insecticides could be expected, if there are deleterious effects on fitness or fitness costs of resistance factors, which may cause decreases in frequency of resistance factors within a population under no insecticide conditions [20].

Chromosomal analyses, using chromosome-substituted lines between the resistant inbred line \#609-10 and the susceptible inbred line \#451-4, revealed that chromosome III of the resistant \#609-10 alone had significant effects on resistance to the three organophosphates [3], whose mechanism was revealed as a mutated AChE, according to its $\sim 15$ times higher $\mathrm{I}_{50}$ value for AChE to fenitroxon than that of the susceptible line [4]. In addition, comparisons of fitnesses showed that the line with chromosome III from the resistant \#609-10 had the lower individual-based intrinsic rate of natural increase (an appropriate fitness measure under density-independent conditions [21]) [5] [6] and the lower population projection trajectory (Figure 3 and Figure 4; [1] [2]) than the line with that from the susceptible \#451-4, suggesting that chromosome III from the resistant \#609-10 had a deleterious effect on fitness under the density-independent condition. Therefore, it is expected that the resistance factor on chromosome III, the resistant-type AChE, tends to decrease in frequency under the density-independent and no insecticide condition, explaining the seasonal increase in susceptibility to organophosphates in the fall, when the Katsunuma population of D. melanogaster increased drastically on the masses of squeezed grapes dumped there.

It should be noted that the susceptible line \#451-4 showed slightly higher resistance levels than \#451-10, the other susceptible inbred line used for constructing chromosome-substituted lines together with \#1465-5 [17]. Therefore, it might be possible that \#451-4 had a resistance factor with some effects for organophosphates; however, compared to \#609-10, \#451-4 still showed lower levels of resistance to organophosphates [3], so that \#451-4 should be considered as a 


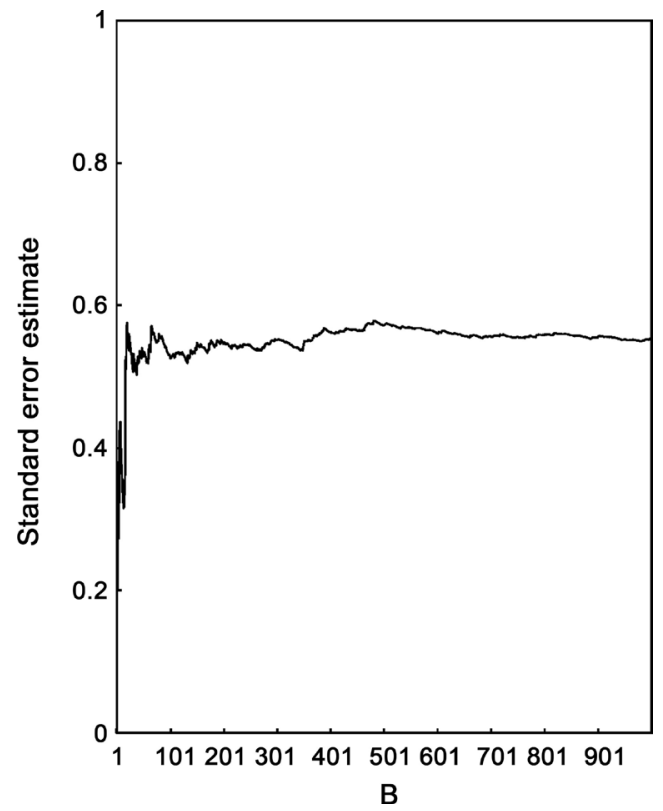

Figure 3. The bootstrap estimate of standard error for the $\log _{10}$-transformed ratio at Day 100 of resistant individuals to susceptible individuals (-1.65), based on density-independent population projection trajectories of individuals with characteristics of chromosomesubstituted lines with the resistant-type AChE and with susceptible-type AChE. A run of 1000 bootstrap replications provided the bootstrap estimate of standard error, 0.5526 . Detailed information can be found in [1].

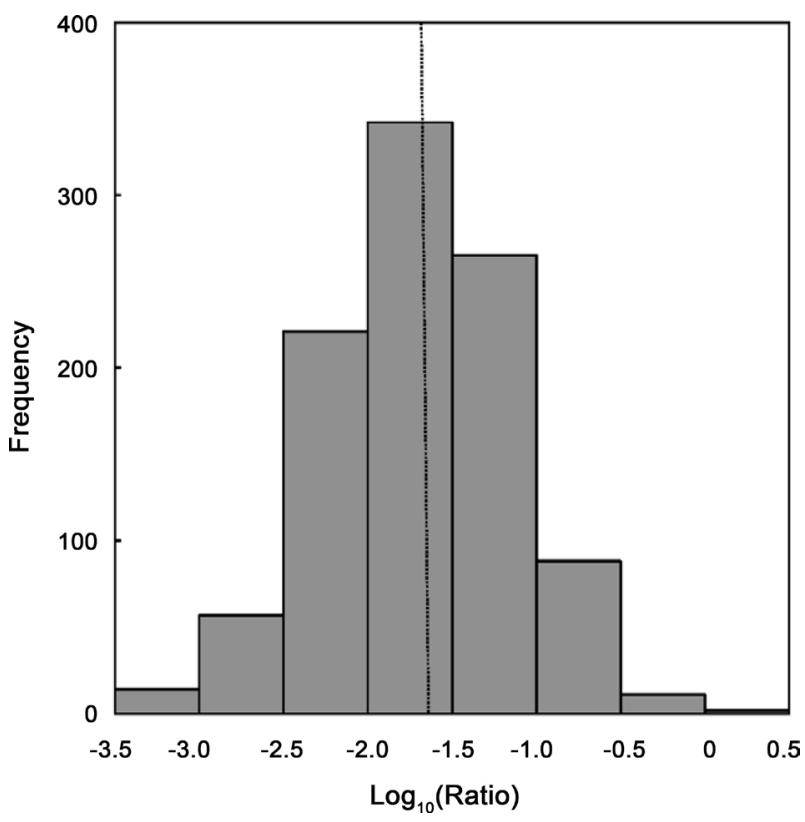

Figure 4. The distribution of 1000 bootstrap replications of the $\log _{10}$-transformed ratio at Day 100 of resistant individuals to susceptible individuals, based on density-independent population projection trajectories of individuals with characteristics of chromosomesubstituted lines with the resistant-type AChE and with susceptible-type AChE. The broken line indicates the mean of the 1000 bootstrap replications $(-1.6948)$. Because the estimate based on the observed data was -1.6522 , and because the bootstrap estimate of standard error was 0.5526 , the bootstrap bias estimate $(-0.0426)$ was reasonably small. Detailed information can be found in [1]. 
susceptible line. This is why we have insisted that insecticide resistance should be considered as a quantitative trait, rather than a qualitative trait [22]. More importantly, comparisons of fitness were conducted using the chromosomesubstituted lines which shared the susceptible chromosomes except chromosome III, where the resistant-type AChE was located. Therefore, the deleterious effect on the individual-based intrinsic rate of increase can be attributable solely to chromosome III of the resistant line \#609-10. In addition, \#609-10 and \#451-4 were both derived from females collected at Katsunuma on the same day, July 1997, so that these lines should be used to elucidate the dynamics of genetic variation in susceptibility to organophosphates within the Katsunuma population, because they actually constructed genetic variation in resistance to organophosphates there at that time (i.e., they were not from different populations, but came from the same population).

\subsection{Measuring Fitness Costs}

Until now, enormous studies have been published, in which fitness costs of insecticide resistance were estimated using different approaches, which controlled effects of genetic background to a varying extent [23]. According to [24], these approaches may be classified into three main categories: a) the "between-population" approach, which simply compares fitness between a resistant field population and a standard laboratory population without controlling the effects of genetic backgrounds on fitness or fitness components; (b) the "experimental selectionbased" approach, which involves repeated selection for resistance in susceptible population to produce matched pairs of resistant-selected populations and susceptible original population, and comparisons of fitness between them; and (c) the "introgression" approach, which involves serial backcrosses of a resistant population with a susceptible population and re-selection for resistance after each cross. Although the introgression-based approach has been considered the most rigorous, this method has been used only rarely in insect evolutionary ecological experiments [24]. In our studies, comparisons of fitness were conducted using chromosome-substituted lines which shared the susceptible chromosomes except chromosome III, on which the resistant-type AChE was located [4]. In addition, the original resistant and susceptible lines were both derived from females collected at Katsunuma on the same day, so that they were considered to have constructed a part of actual genetic variation in resistance to organophosphates there at that time [3]. Therefore, we believe that our experimental approach to estimate deleterious effects of the resistance factor was consistent well with a majority of other fitness cost experiments published to date.

\subsection{Measuring the Intrinsic Rate of Natural Increase at the Genotypic (Individual) Level}

In the earlier sections, I have briefly described our research and explained why we conducted these studies. However, Lawo and Lawo [8] argued that it was a 
misconception to apply the intrinsic rate of natural increase to individuals, because it is a population parameter. We consider that their criticisms were incorrect for the following reasons.

1) As discussed by Charlesworth [21], population genetics models show that the intrinsic rate of natural increase, $r_{i j}$, defined as the real root of the EulerLotka equation $\sum_{x} e^{-r_{i j} x} k_{i j}(x)=1$, is an appropriate measure of fitness for a diploid genotype $i j$ under density-independent conditions under many circumstances (p. 178). Here, $k_{i j}(x)$ is the reproductive function, the net expectation of female offspring produced by a female aged $x$, weighted by the probability of survival from the zygote stage. Fitness is usually defined for individuals, unless group selection is being considered. Of course, group selection theories may be relevant for traits such as altruistic behaviors but no one would probably consider that this is appropriate for insecticide resistance within a natural population of $D$. melanogaster. If it is acceptable that fitness should be defined for individuals, our attempt to measure the intrinsic rate of natural increase at the genotype (individual) level is a reasonable approach.

2) As this argument shows, the intrinsic rate of natural increase is an appropriate measure of genotypic fitness under density-independent conditions [21]. If we try to construct density-independent conditions in the laboratory, populations of organisms with high fecundity, such as D. melanogaster, would need to be started from small population sizes. The most efficient way to ensure densityindependence would be to construct a population with a single male-female pair of a known genotype. If we started experimental populations from larger population sizes in order to obtain intrinsic rates of natural increase, confounding factors, such as density-dependence, would have been involved in the obtained results.

3) If there is variation in intrinsic rate of natural increase among genotypes within a population (genetically heterogeneous population), the intrinsic rate of natural increase for the population as a whole would change with time, because the frequencies of genotypes within the population change with time. In other words, the intrinsic rates of natural increase of the population as a whole should be dependent on the intrinsic rates of natural increase of the genotypes and the frequencies of the genotypes involved in the population. Therefore, the intrinsic rate of natural increase ultimately needs to be defined at the genotypic (individual) level.

\section{Conclusion}

As I have explained earlier, the purpose of our research was not merely to measure intrinsic rates of natural increase for several populations and compare them among the populations but to obtain insights into how the frequencies of resistant genotypes and resistance genes would change within the Japanese local population of D. melanogaster during population growth that we considered the most important ecological factor there, which could explain the observed reduction in 
the levels of resistance to organophosphates within the natural population [7]. Therefore, we made new attempts from both theoretical and experimental standpoints, which had never been paid attention in the research field of insecticide resistance before, and not necessarily consistent with other research which just tried to measure intrinsic rates of natural increase. This paper should clarify the purpose of our work. I hope our endeavors made in a series of studies and criticisms on our work may contribute to future research on insecticide resistance in a constructive way. In addition, the concept of the genotypic intrinsic rate of natural increase should be important and valuable especially when we need to consider the fluctuations in genetic variation within not only growing populations but also shrinking populations, such as the graying Japanese population (cf. [25]).

\section{Acknowledgements}

I thank Professor Brian Charlesworth (Univ. of Edinburgh) for valuable comments and suggestions on the manuscript. I am grateful to John-Philip Lawo for his criticisms and discussion on our research. This article was written after direct correspondence via e-mail with John-Philip Lawo.

\section{References}

[1] Miyo, T. (2011) Population Model of Fluctuations in Organophosphate Resistance of Drosophila melanogaster. Roles of a Mutated Acetylcholinesterase and a Cytochrome P450. Russian Journal of Ecology, 42, 510-517.

https://doi.org/10.1134/S1067413611060178

[2] Miyo, T. and Charlesworth, B. (2004) Density-Independent Population Projection Trajectories of Chromosome-Substituted Lines Resistant and Susceptible to Organophosphate Insecticides in Drosophila melanogaster. BMC Genetics, 5, 31. https://doi.org/10.1186/1471-2156-5-31

[3] Miyo, T. and Oguma, Y. (2002) Negative Correlations between Resistance to Three Organophosphate Insecticides and Productivity within a Natural Population of Drosophila melanogaster (Diptera: Drosophilidae). Journal of Economic Entomology, 95, 1229-1238. https://doi.org/10.1603/0022-0493-95.6.1229

[4] Miyo, T. and Oguma, Y. (2010) Contributions of Three-Site Mutations in Acetylcholinesterase and Cytochrome P450 to Genetic Variation in Susceptibility to Organophosphate Insecticides within a Natural Population of Drosophila melanogas ter. Population Ecology, 52, 159-169. https://doi.org/10.1007/s10144-009-0157-1

[5] Miyo, T., Oguma, Y. and Charlesworth, B. (2003) The Comparison of Intrinsic Rates of Increase among Chromosome-Substituted Lines Resistant and Susceptible to Organophosphate Insecticides in Drosophila melanogaster. Genes and Genetic Systems, 78, 373-382. https://doi.org/10.1266/ggs.78.373

[6] Miyo, T., Oguma, Y. and Charlesworth, B. (2006) Seasonal Fluctuation in Susceptibility to Insecticides within Natural Populations of Drosophila melanogaster. II. Features of Genetic Variation in Susceptibility to Organophosphate Insecticides within Natural Populations of D. melanogaster. Genes and Genetic Systems, 81, 273-285. https://doi.org/10.1266/ggs.81.273

[7] Miyo, T., Akai, S. and Oguma, Y. (2000) Seasonal Fluctuation in Susceptibility to 
Insecticides within Natural Populations of Drosophila melanogaster. Empirical Observations of Fitness Costs of Insecticide Resistance. Genes and Genetic Systems, 75, 97-104. https://doi.org/10.1266/ggs.75.97

[8] Lawo, J.-P. and Lawo, N.C. (2011) Misconceptions about the Comparison of Intrinsic Rates of Natural Increase. Journal of Applied Entomology, 135, 715-725. https://doi.org/10.1111/j.1439-0418.2011.01608.x

[9] Wilson, T.G. (1988) Drosophila melanogaster (Diptera: Drosophilidae): A Model Insect for Insecticide Resistance Studies. Journal of Economic Entomology, 81, 22-27. https://doi.org/10.1093/jee/81.1.22

[10] Miyo, T., Takamori, H., Kono, Y. and Oguma, Y. (2001) Genetic Variation and Correlations among Responses to Five Insecticides within Natural Populations of Drosophila melanogaster (Diptera: Drosophilidae). Journal of Economic Entomology, 94, 223-232. https://doi.org/10.1603/0022-0493-94.1.223

[11] Hatano, Y., Inoue, Y., Watada, M., Akai, S.M., Watanabe, T.K., Yamamoto, M.-T., Hirai, K., Takegawa, H. and Itoh, M. (1999) Further Genetic Studies on the Katsunuma Population of Drosophila melanogaster. Genes and Genetic Systems, 74, 219-225. https://doi.org/10.1266/ggs.74.219

[12] David, J. R., Gibert, P., Legout, H., Pétavy, G., Capy, P. and Moreteau, B. (2005) Isofemale Lines in Drosophila: An Empirical Approach to Quantitative Trait Analysis in Natural Populations. Heredity, 94, 3-12. https://doi.org/10.1038/sj.hdy.6800562

[13] Mukai, T. (1964) The Genetic Structure of Natural Populations of Drosophila melanogaster. I. Spontaneous Mutation Rate of polygenes Controlling Viability. Genetics, 50, 1-19.

[14] Miyo, T. (2012) The Genetic Architecture of Insecticide Resistance within a Natural Population of Drosophila melanogaster. Open Journal of Genetics, 2, 90-94. https://doi.org/10.4236/ojgen.2012.22013

[15] Abbott, W.S. (1925) A Method of Computing the Effectiveness of an Insecticide. Journal of Economic Entomology, 18, 265-267. https://doi.org/10.1093/jee/18.2.265a

[16] Sokal, R.R. and Rohlf, F.J. (1995) Biometry. 3rd Edition, Freeman, New York.

[17] Miyo, T., Kono, Y. and Oguma, Y. (2002) Genetic Basis of Cross-Resistance to Three Organophosphate Insecticides in Drosophila melanogaster (Diptera: Drosophilidae). Journal of Economic Entomology, 95, 871-877. https://doi.org/10.1093/jee/95.5.871

[18] Morton, R.A. (1993) Evolution of Drosophila Insecticide Resistance. Genome, 36, 1-7. https://doi.org/10.1139/g93-001

[19] Charlesworth, B. and Charlesworth, D. (2010) Elements of Evolutionary Genetics. Roberts and Company Publishers, Greenwood Village.

[20] Crow, J.F. (1957) Genetics of Insect Resistance to Chemicals. Annual Review of Entomology, 2, 227-246. https://doi.org/10.1146/annurev.en.02.010157.001303

[21] Charlesworth, B. (1994) Evolution in Age-Structured Populations. 2nd Edition, Cambridge University Press, Cambridge. https://doi.org/10.1017/CBO9780511525711

[22] Miyo, T., Keil, C.B.O., Hough-Goldstein, J.A. and Oguma, Y. (1999) Inheritance of Resistance to Esfenvalerate in Colorado Potato Beetles (Coleoptera: Chrysomelidae). Journal of Economic Entomology, 92, 1031-1038. https://doi.org/10.1093/jee/92.5.1031 
[23] Kliot, A. and Ghanim, M. (2012) Fitness Costs Associated with Insecticide Resistance. Pest Management Science, 68, 1431-1437. https://doi.org/10.1002/ps.3395

[24] Raymond, B., Wright, D.J. and Bonsall, M.B. (2011) Effects of Host Plant and Genetic Background on the Fitness Costs of Resistance to Bacillus thuringiensis. Heredity, 106, 281-288. https://doi.org/10.1038/hdy.2010.65

[25] Miyo, T. (2017) Why Do We Care for Old Parents? Evolutionary Genetic Model of Elderly Caring. Open Journal of Genetics, 7, 20-39.

https://doi.org/10.4236/ojgen.2017.71003 